\title{
Les odontomes complexes des maxillaires : à propos d'un cas clinique mandibulaire compliqué d'une ostéite
}

\section{RÉSUMÉ}

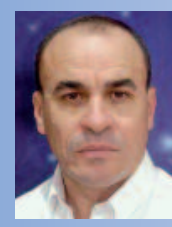

Hamid BOUKAIS

Service de pathologie et chirurgie buccale, CHU Béni-Méssous, Alger,

Faculté de médecine d'Alger, Algérie.

Wacila ZERROUKI

Service de pathologie et chirurgie buccale, CHU Béni-Méssous, Alger.

Chafika BABA-MEHDID

Service d'odontologie conservatrice CHU Béni-Méssous, Alger.

\section{Rachida AOUAMEUR}

Service d'anesthésie-réanimation, CHU Béni-Méssous, Alger.

\section{Nadjah OUSSALEM}

Chirurgien-dentiste spécialiste,

exercice libéral

Tizi-Ouzou, Algérie.

Assia SLIMANI

Service d'anatomie pathologique, CHU Béni-Méssous, Alger.

\section{Assia HENNI}

Service d'anatomie pathologique, CHU Béni-Méssous, Alger.

\section{Hocine SKLAB}

Service d'anatomie pathologique, CHU Béni-Méssous, Alger.

Kheira BOUZID-BENDISSARI Service d'anatomie pathologique, CHU Béni-Méssous, Alger.

\section{Introduction}

Les odontomes intra-osseux des maxillaires peuvent rester longtemps asymptomatiques, ou être à l'origine d'une infection (cellulite ou ostéite) et s'extérioriser dans la cavité buccale. Dans de rares situations, ces tumeurs peuvent s'extérioriser par tentative d'éruption.

\section{Matériel et méthodes}

Étude d'un cas clinique.

\section{Résultats}

Une patiente, âgée de 62 ans, a consulté pour une infection mandibulaire récidivante. Les explorations cliniques, radiologiques et anatomopathologiques ont permis de découvrir un odontome complexe intramandibulaire, compliqué d'une ostéite.

\section{Conclusion}

Des cas similaires ont été rapportés dans la littérature mondiale. L'infection se produit lorsque l'odontome tente de faire éruption dans la cavité buccale. Devant une infection récidivante dans un secteur édenté de l'arcade, une exploration radiologique doit toujours être réalisée pour étayer le diagnostic positif et surtout étiologique.

tumeurs odontogènes

- ostéites

- odontomes complexes

- éruption
AOS 2011;254:153-162

DOI: $10.1051 /$ aos/2011207

(C) AEOS / EDP Sciences 


\section{Introduction}

$>$

Les odontomes sont des tumeurs parmi les tumeurs odontogéniques bénignes les plus fréquentes des maxillaires, de découverte le plus souvent fortuite à l'occasion d'un examen radiographique de routine. Ces néoformations sont constituées d'émail, de dentine, de cément et de pulpe disposés anarchiquement au sein de la structure osseuse mandibulaire le plus souvent ou maxillaire. Deux formes radiocliniques sont décrites :

- les odontomes complexes, faits d'une masse constituée d'un assemblage anarchiquement disposé, de tissus minéralisés (émail, dentine, cément) et de pulpe dentaire ;
- les odontomes composés, constitués d'un ensemble de petites dents rudimentaires, assemblées en amas.

Ces tumeurs peuvent rester longtemps asymptomatiques. Elles peuvent en revanche, dans certains cas, entraîner des manifestations cliniques telles que douleurs, infections, fistules et extériorisation. L'indication de leur exérèse chirurgicale s'impose alors.

À travers cette publication, nous rapporterons un cas clinique d'odontome complexe géant intramandibulaire chez une patiente d'âge adulte, traité comme un processus infectieux récidivant et fistulisé, malgré la prescription d'une série de traitements antibiotiques.

\section{Revue de la littérature. Historique}

Au début de leur découverte, les odontomes étaient considérés comme des tumeurs vraies. Mais très vite, cette appellation a disparu pour être remplacée par la notion de malformations de type hamartomateux [1-2].

En effet, depuis les travaux de Broca en 1866 [3], le terme d'odontome est réservé à un nombre plus restrictif de lésions dans lesquelles les odontoblastes et les améloblastes sont normalement formés et produisent de l'émail et de la dentine ; mais l'organisation de ces tissus en organes dentaires échoue ; apparaissent alors des formations anormales ressemblant ou non à des dents.

Globalement, et jusqu'à I'heure actuelle, l'étiopathogénie des odontomes reste encore mal élucidée. La notion d'hérédité est évo- quée par certains auteurs. L'anomalie est transmise selon un mode autosomique dominant, une mutation génétique serait également une étiologie probable de cette transmission [4].

La classification des odontomes a subi et subit encore des modifications. Les appellations et les regroupements de ces pathologies diffèrent d'un auteur à un autre.

À coté des formes classiques d'odontomes composés et complexes et d'odonto-améloblastomes, la littérature décrit des cas de formes exceptionnelles comme I'odontomatose quand les odontomes sont multiples, l'odontome kystique ou kyste odontogène calcifié, qui évolue à l'intérieur d'une cavité kystique ou de sa membrane, les odontomes 
géminés, les odontomes composites inclus, gestants ou ectasiants [5].

La classification actuelle des tumeurs odontogéniques est faite selon la nature épithéliale, mésenchymateuse ou mixte (épithélio-mésenchymateuse) du tissu qui leur a donné naissance. Les odontomes sont des tumeurs mixtes odontogènes des maxillaires puisqu'elles ont à la fois une origine épithéliale et conjonctive [6-9].

La découverte d'un odontome dans un secteur d'un maxillaire doit toujours faire rechercher d'autres localisations homo- ou controlatérales, maxillaires ou mandibulaires. Lorsque leurs localisations sont multiples, ils peuvent s'inscrire dans le tableau d'un syndrome (odontomatose) qui associe en plus des odontomes : des dents invaginées; des dents gémi-

\section{Observation clinique}

Madame A. F., âgée de 62 ans, originaire et demeurant à Tizi Ouzou (Algérie), a consulté en mai 2010, pour tuméfactions mandibulaires gauches récidivantes malgré plusieurs prescriptions antibiotiques. La répétition des poussées infectieuses a conduit à l'exploration radiologique qui a mis en évidence une image pathologique, à la suite de quoi la patiente fut orientée dans le service pour un avis spécialisé.

À l'interrogatoire, la patiente décrit des tuméfactions récidivantes douloureuses à la palpation, accompagnées d'une rougeur cutanée et d'une hypoesthésie labio-mentonnière. Elle rapporta aussi la notion d'écoulement de liquide dans la bouche.

L'examen clinique maxillo-facial a retrouvé une asymétrie faciale due à la présence d'une tumé- nées, une sténose œsophagienne et une cirrhose interstitielle chronique du foie [10-12]. Plusieurs cas ou des séries de cas d'odontomes ont été rapportés dans la littérature [13-18]. Dans ces publications, les auteurs s'accordent sur un certain nombre de caractéristiques cliniques, radiologiques et évolutives de ces pathologies, comme leur découverte souvent fortuite, leur origine embryologique, leur aspect radiologique hyperdense, une surinfection possible avec ou sans fistulisation et leur bénignité absolue. Des cas d'odontomes extériorisés ou infectés après une longue phase asymptomatique ont été rapportés. L'extériorisation et par conséquent la surinfection seraient consécutives à la résorption osseuse engendrée par la mise en place d'une prothèse dentaire amovible [19-23].

faction discrète génienne basse gauche. La palpation des aires ganglionnaires a mis en évidence une adénopathie mobile sous-angulomaxillaire homolatérale. L'examen de la cavité buccale retrouve une fistule productive de sérosité et de pus, une induration et un comblement du vestibule postéro-latéral gauche. À travers la fistule, nous notons la présence d'un fragment de tissu dentaire de couleur jaunâtre évoquant l'aspect du cément (fig. 1).

À l'examen du panoramique dentaire (fig. 2), nous avons retrouvé une image radio-opaque à grand axe antéropostérieur, située au niveau de la branche horizontale gauche de la mandibule, faite de l'assemblage de plages de tissus dont la densité est de type dentaire. Cette image, bordée à sa partie basale et latérale par 


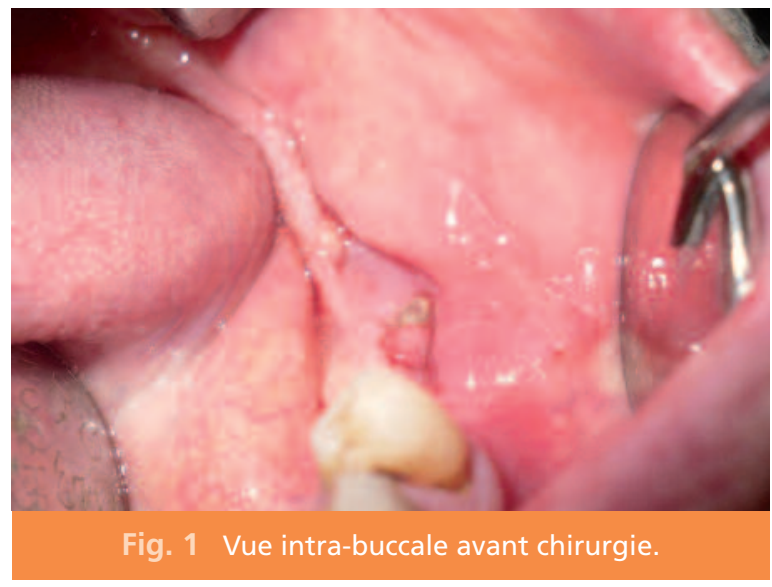

un anneau radio-claire, s'étend sur $8 \mathrm{~cm}$ postérieurement à la première prémolaire (34). En bas, l'image arrive jusqu'au contact du canal alvéolaire inférieur, dont l'intégrité semble préservée.

Par ailleurs, ce cliché a permis la découverte fortuite de deux autres images radiologiques similaires, de volume moindre, l'une dans la région molaire inférieure droite et l'autre au niveau de la région molaire supérieure droite. Aucune symptomatologie n'a été rapportée par la patiente au niveau de ces deux régions. Nous retrouvons aussi des racines dentaires résiduelles au niveau molaire supérieur gauche.

La conduite thérapeutique a consisté dans un premier temps en une antibiothérapie empirique faite d'une association d'amoxicilline et de métronidazole, associée à un antalgique et un antiseptique buccal. Un drainage par voie fistulaire et pression manuelle au niveau de la partie comblée du vestibule a été effectué.

La patiente revue une semaine après a présenté une évolution favorable. Une chirurgie type énucléation-curetage fut alors programmée un mois après la première consultation, après refroidissement par antibiothérapie.

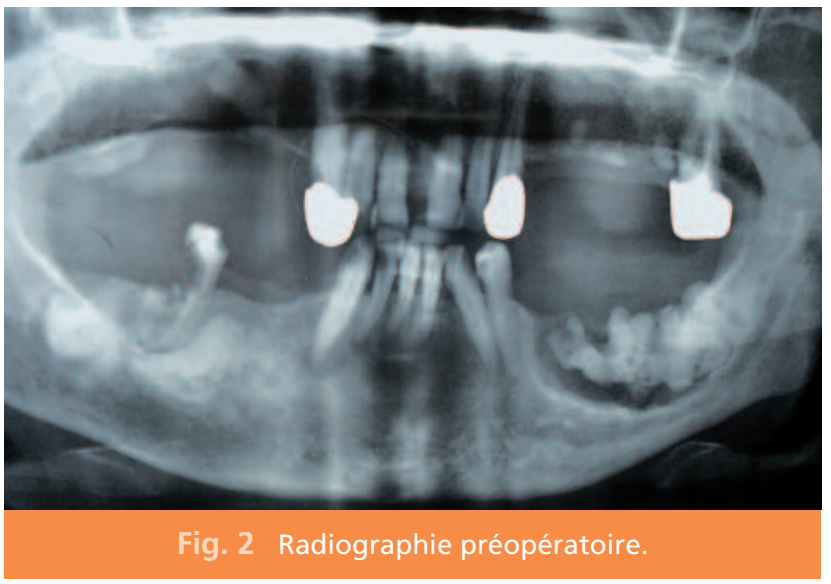

L'intervention s'est déroulée en plusieurs temps sous anesthésie loco-régionale. Après asepsie de la cavité buccale, par bain de bouche à la chorhéxidine, l'incision a concerné la crête, complétée par une incision de décharge vestibulaire. Le décollement de proche en proche d'un lambeau muco-périosté a permis d'entamer le clivage facile d'une masse de consistance dure d'environ $6 \mathrm{~cm}$ de diamètre, faite sur le plan macroscopique de tissus dentaires fusionnés et disposés en amas (cément, dentine). À cette masse est accolé un fragment d'os d'aspect nécrotique et infecté, rappelant l'aspect d'un séquestre. La cavité opératoire a été alors curetée et soigneusement rincée au sérum, les lambeaux ont été remis en place et suturés. La pièce opératoire a été fixée et envoyée pour étude anatomopathologique.

Un traitement antibiotique, antalgique et des bains de bouche ont été prescrits. Une semaine après, l'ablation des fils de suture a été réalisée (fig. 3 à 6).

L'étude anatomopathologique de la pièce opératoire après décalcification met en évidence une structure amorphe polycyclique acellulaire faiblement éosinophile rappelant la structure 

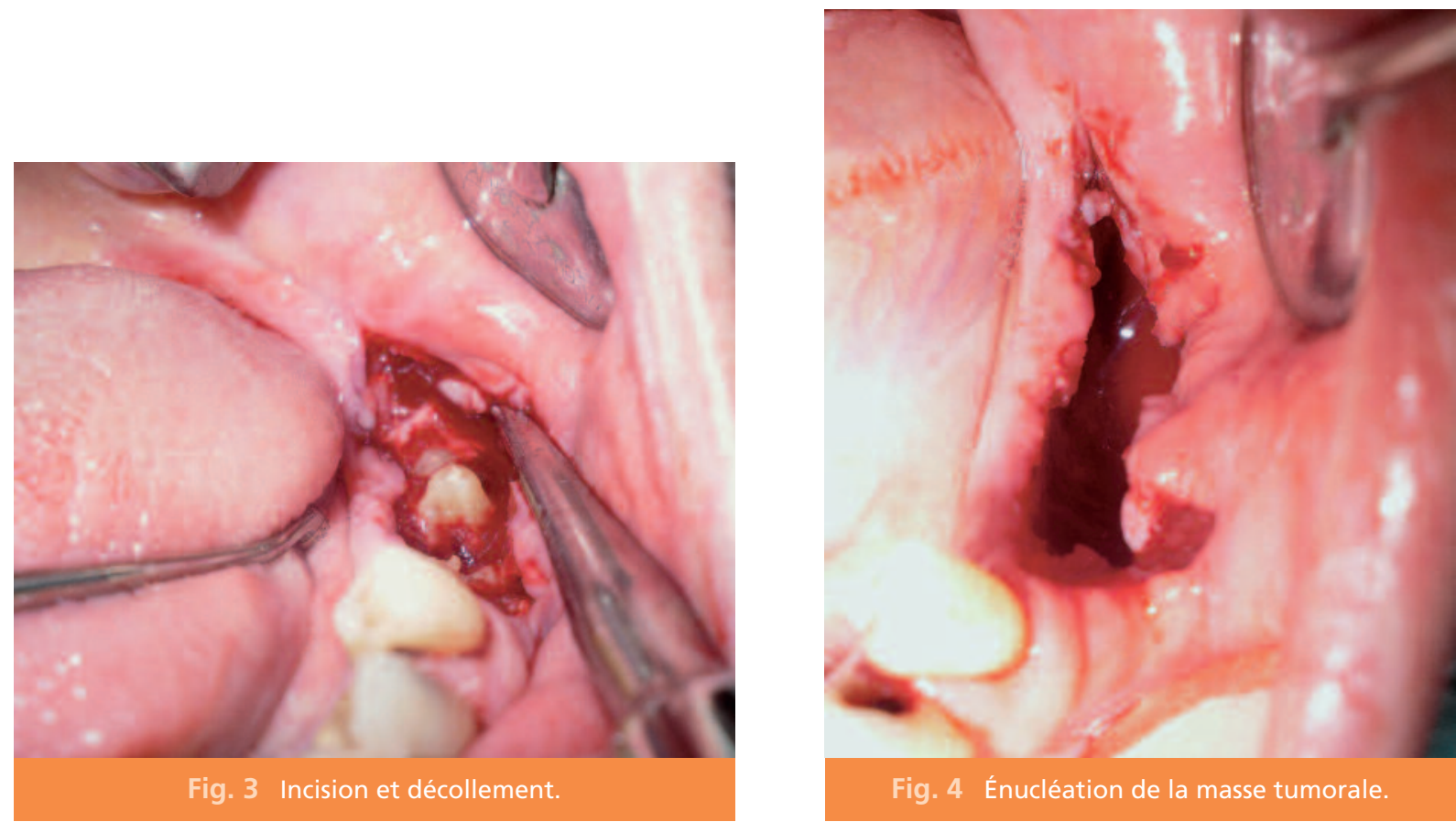

Fig. 4 Énucléation de la masse tumorale.
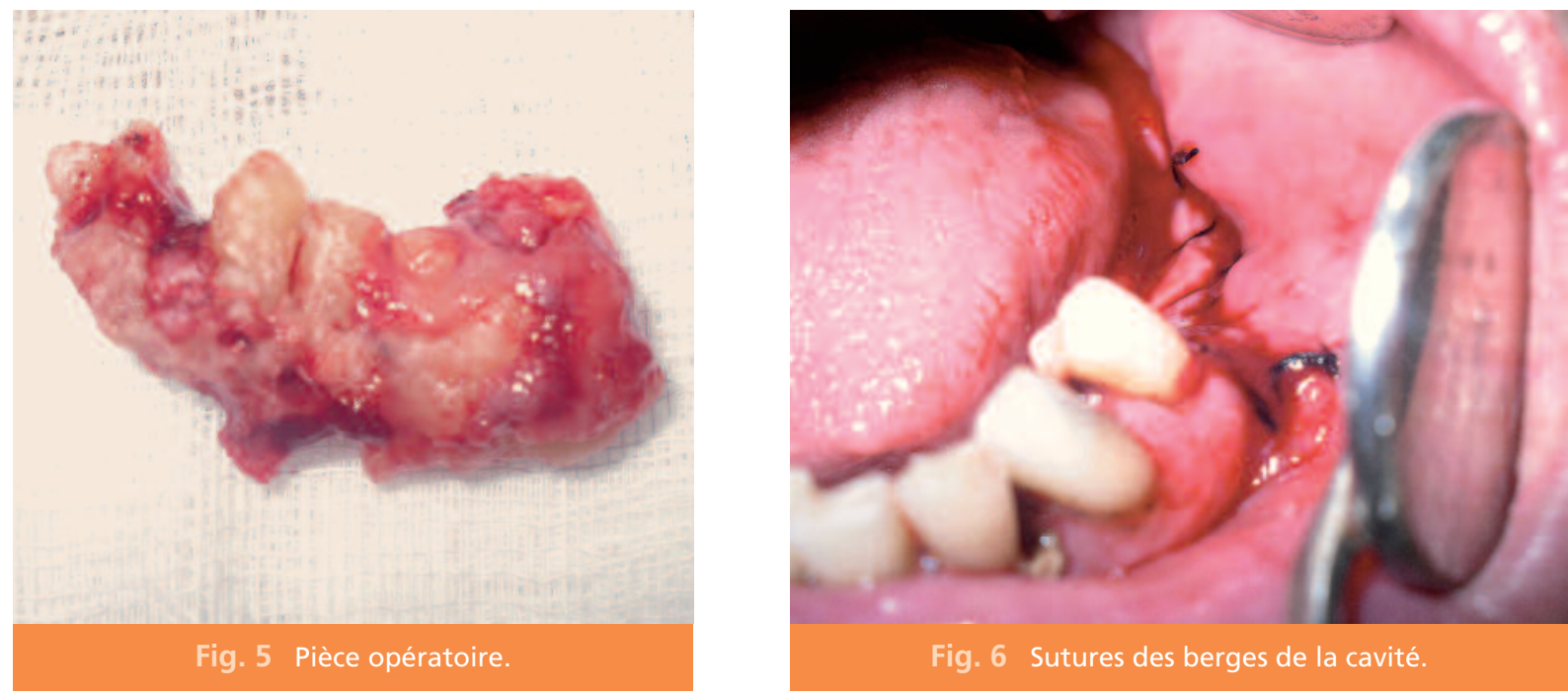

du tissu dentigène (fig. 7). À un fort grossissement, l'aspect anatomopathologique est fait d'une densification centrale, rappelant la structure du cément (fig. 8).

Le contrôle radiologique, effectué à 21 jours après l'intervention (fig. 9), met en évidence une réparation en cours de la cavité osseuse opératoire. À 2 mois, la réparation osseuse est plus avancée (fig. 10). La patiente est encore suivie pour des contrôles périodiques.

Nous notons une amélioration de l'hypoesthésie labio-mentonnière. Un contrôle radiologique à 6 mois après la chirurgie a été réalisé et montre une réparation osseuse plus avancée (fig. 11). 


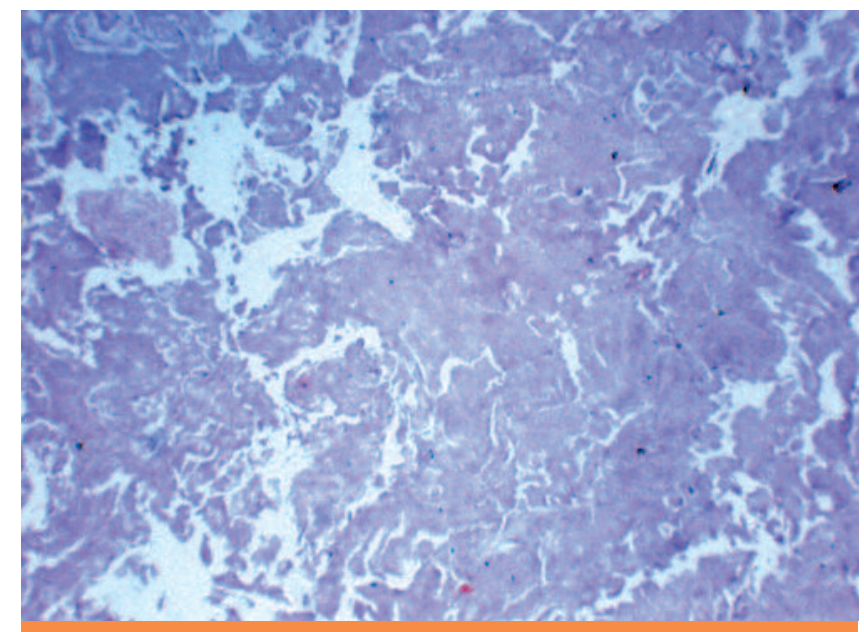

Fig. 7 Structure anatomopathologique (grossissement x 4).

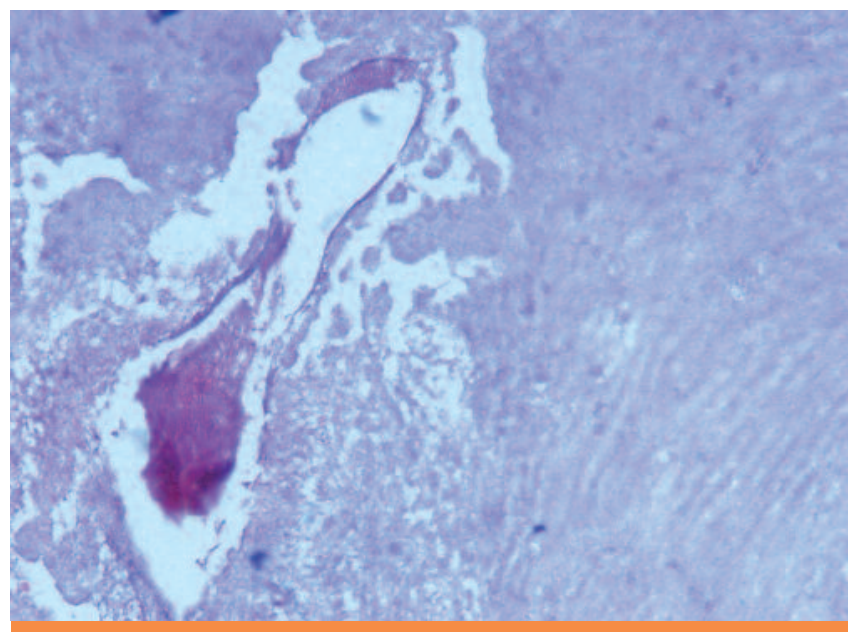

Fig. 8 Structure anatomopathologique (grossissement x 20).

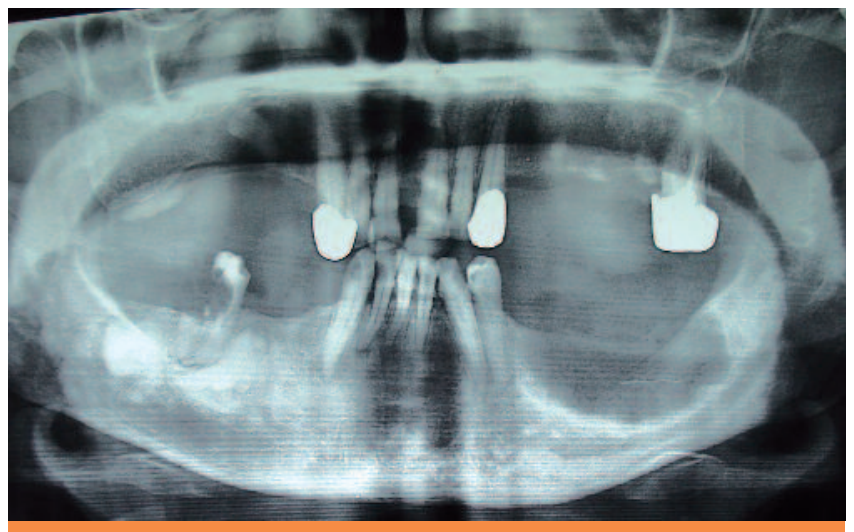

Fig. 9 Contrôle radiologique à 3 semaines.

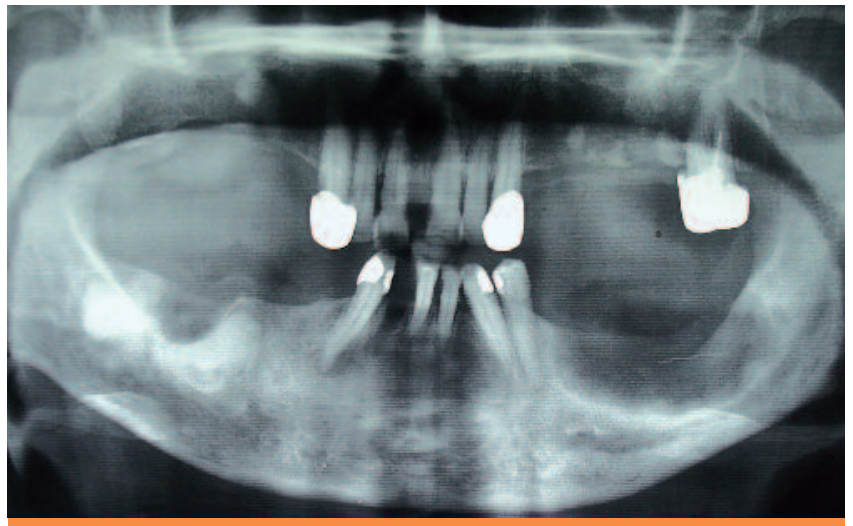

Fig: 10 Contrôle radiologique à 2 mois.

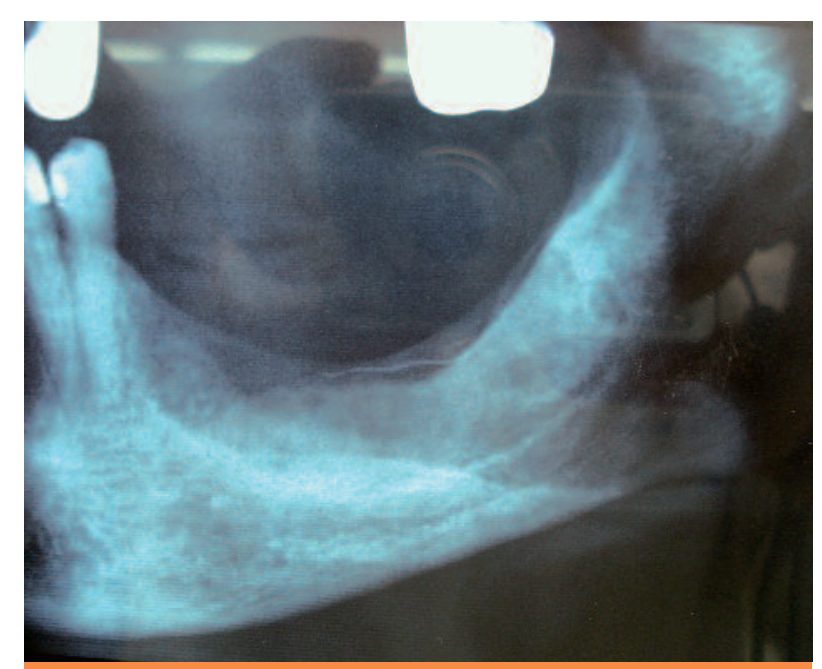

Fig. 11 Contrôle radiologique à 6 mois. 


\section{Discussion}

Les odontomes sont des tumeurs qui affectent plus souvent le sujet jeune. La persistance anormale d'une dent lactéale ou l'absence d'éruption d'une dent définitive à un âge où elle devrait être sur l'arcade doivent motiver la consultation et permettre la découverte de ces malformations qui font obstacle à la mise en place physiologique spontanée d'une ou de tout un groupe de dents [24]. Chez l'adulte, la surinfection ou l'extériorisation de l'odontome longtemps asymptomatique sont l'un des motifs de la consultation.

Hidalgo-Sanchez et ses collaborateurs [25], dans leur méta-analyse portant sur 3065 cas d'odontomes à travers la revue de trente articles publiés ces dernières années dans la littérature, ne montrent pas une prédominance de ces tumeurs selon le sexe ; ils sont, pour la plupart diagnostiqués lors de la $2^{\mathrm{e}}$ décennie. Le siège de prédilection est le maxillaire supérieur dans sa région antérieure. La même analyse montre que les odontomes composés sont plus fréquents que les odontomes complexes. Ces tumeurs, souvent asymptomatiques, peuvent être découvertes à l'occasion d'un examen radiologique de routine.

Si l'on discute les caractéristiques des odontomes complexes décrites à travers les résultats de cette méta-analyse, avec celles de notre cas clinique, nous pouvons conclure sur quelques différences en rapport avec l'âge, le siège et la situation postérieure de la tumeur. Ce cas clinique est en concordance avec les cas présentés dans la littérature, seulement en ce qui concerne la phase clinique asymptomatique qui peut être relativement longue [6, 8, 9, 26 27].
Le cas clinique que nous présentons se caractérise, comme dans les données de HidalgoSanchez et al. [25], par une longue phase de latence asymptomatique.

Or, dans le cas présent, il s'agit d'un odontome complexe de localisation mandibulaire, compliqué d'accidents aigus itératifs diagnostiqués et traités comme accidents cellulitiques, récidivant en l'absence de traitement étiologique, c'est-à-dire l'exérèse de l'odontome.

La nature mixte de l'image, à savoir la présence d'un matériel radio-opaque à tonalité dentaire comportant quelques travées de radio-clarté, peut orienter le diagnostic, la certitude étant du ressort de l'analyse anatomopathologique.

La constatation d'un hiatus entre I'os mandibulaire d'apparence sain et la lésion radioopaque principale peut évoquer le diagnostic d'odonto-améoblastome puisque Bouchet et al [28] ont rapporté le cas d'un kyste odontogène calcifié associé à un odontome complexe. L'odonto-améloblastome est une néoformation caractérisée par la présence de structures organoïdes d'aspect comparable à celui de l'odontome avec en plus une prolifération épithéliale de nature améloblastique. Le fibro-odontome améloblastique et le fibrome améloblastique ont des structures anatomopathologiques qui se ressemblent, mais la dentine n'est pas retrouvée dans la structure de ce dernier. II est de ce fait indispensable de discuter toutes ces tumeurs avant d'avoir la certitude anatomopathologique [7, 12, 14, 29, 30, 31].

La chirurgie de ces tumeurs fait appel à l'énucléation-curetage. L'énucléation devient difficile, 
lorsque les limites de la tumeur sont intimement intriquées avec le tissu sain voisin. Lorsque l'énucléation est totale, la récidive est exceptionnelle. En peropératoire, lorsque la tumeur est de siège mandibulaire et très étendue vers le rebord basilaire, la survenue de fracture est possible.

\section{Conclusion}

Les odontomes représentent une entité importante parmi les tumeurs odontogéniques bénignes des maxillaires. Bien qu'elles soient asymptomatiques dans la grande majorité des cas, ces pathologies peuvent être à l'origine de manifestations infectieuses, de névralgies et parfois d'hypoesthésie par phénomène de compression des éléments nerveux.

Le traitement de ces tumeurs est purement chirurgical, mais précédé d'une antibiothérapie en cas d'infection. Ces tumeurs ne récidivent jamais lorsque leur exérèse chirurgicale a été
Le diagnostic anatomopathologique, toujours fait après décalcification de la pièce opératoire, est basé sur la mise en évidence de plages de tissu dentaire (dentine, émail, cément), mal organisé et anarchiquement disposé au sein de la trame osseuse [30].

complète. L'énucléation tumorale par clivage est la technique la plus appropriée pour leur traitement, la présence d'un halot radiologique radio-claire périphérique facilite l'énucléaction en bloc de la tumeur.

Devant toute infection ou fistule traînante de la cavité buccale, une exploration radiologique est indispensable. Celle-ci peut mettre en évidence un foyer infectieux (racine incluse, réaction kystique ou granulomateuse), mais parfois des pathologies tumorales jusque-là méconnues.

\section{Bibliographie}

1. Pindborg JJ, Kramer IRH, Torloni $\mathrm{H}$. Histological typing of odontogenic tumors, Jaw cysts, and allied lesions. International Histological Classification of tumors, $\mathrm{N}^{\circ} 5$ World Health Organisation, Geneva, 1971.

2. Shafer WG, Hine MK, Levy BM.

A textbook of oral pathology. Philadelphia : Saunders,
1983:1-85,258-316,317-38, 528-93.

3. Broca P. Traité des tumeurs. Paris : Asselin, 1866:350.

4. Hitchin AD. The aetiology of the calcified composite odontoma.

Br Dent J 1971;130: 475-82.

5. Brabant $\mathrm{H}$, Klees $\mathrm{L}$, Werelds $\mathrm{R}$.
Anomalies, mutilations et tumeurs des dents humaines. Paris : Prélat, 1958.

6. Peron JM, Hardy $\mathrm{H}$. Tumeurs odontogéniques mixtes.

Rev Stomatol Chir Maxillofac 2009;110:217-20.

7. Teman G, Lacan A, Suissa M, Sarazin L. Imagerie dento-maxillaire. Encycl Med Chir 
Stomatologie

2009;31-676-A-10.

8. Louryan S, Lemort M. Imagerie de la pathologie buccale. Montpellier : Sauramps Medical, 2007.

9. Martin-Duverneuil N, Jarquin S, Chiras J.

Les tumeurs du massif facial. Encycl Med Chir Radiodiagnostic 2006;31-675-A-20.

10. Herrmann M. Über vom Zahnsystem ausgehende Tumoren bei Kindern.

Fortschr Kiefer Gesichtschir 1958:4:226-9.

11. Schmidseder $R$,

Hausamen JE.

Multiple

odontogenic tumors

and other anomalies.

Oral Surg 1975;39:249-58.

12. Piette $\mathrm{E}$, Reychler $\mathrm{H}$. Traité de pathologies buccale et maxillo-faciales. Bruxelles :

De Boeck-Wesmael, 1991;1181-259.

13. Daley TD, Wysocki GP, Pringle GA.

Relative incidence of odontogenic tumors and oral and jaw cysts in a Canadian population. Oral Surg Oral Med Oral Pathol 1994;77:276-80.

14. Regezzi JA, Kerr DA, Courtney RM.

Odontogenic tumors. Analysis of 706 cases. Oral Surg 1978;36:771-8.

15. Junquera L, Vicente JC, Roig P, Olay $S$, Rodríguez-Recio $\mathrm{O}$.
Intraosseus odontoma erupted into the oral cavity: An unusual pathology. Med Oral Patol Oral Cir Bucal (Ed. impr.) v.10 n.3 Valencia mayo-jul. 2005.

16. Ide F, Shimoyama T, Horie N. Gingival periphera odontoma in an adult: case report. J Periodontol 2000;71:830-2.

17. Rumel $A$, de Freitas $A$, Birman EG, Tannous LA, Chacon PT, Borkas S.

Erupted complex odontoma. Report of a case.

Dentomaxillofac Radiol 1980;9:5-9.

18. Al-Sahhar WF, Putrus ST. Erupted odontoma. Oral Surg Oral Med Oral Pathol 1985;59:225-6.

19. Serio FG, Levy BA. Erupted compound odontoma.

Review and report of case. Ann Dent 1987;46:41-2.

20. Gomel M, Seçkin T. An erupted odontoma: case report. J Oral Maxillofac Surg 1989;47:999-1000.

21. López-Areal L, Silvestre Donat $\mathrm{F}$, Gil Lozano J. Compound odontoma erupting in the mouth: 4-year follow-up of a clinical case. J Oral Pathol Med 1992;21:285-8.

22. Kaneko M, Fukuda M, Sano T, Ohnishi T, Hosokawa Y. Microradiographic and microscopic investigation of a rare case of complex odontoma. Oral Surg Oral Med Oral Pathol Oral Radiol Endod 1998;85:131-4.

23. Ferrer MJ, Silvestre FJ, Estrelles E, Grau D, López R. Recurrent infection of a complex odontoma following eruption in the mouth. Med Oral 2001;6:269-5.

24. Korbendeau JM, Guyomard F.

Diagnostic et traitement des obstacles à l'éruption : dents surnuméraires et odontomes. In: Chirurgie parodontale orthodontique. Paris : CdP, 1998:65-89.

25. Hidalgo-Sanchez O,

Leco-Berrocal MI,

Martinez-Gonzalez JM.

Meta-analysis

of the epidemiology and clinical manifestations of odontomas.

Med Oral Patol Oral Cir Bucal 2008 Nov 1;13(11):

E730-4.

26. School RJ, Kellett HM, Neumann DP, Lurie AG.

Cysts and cystic lesions of the mandible.

Clinical and radiologic-histopathologic review.

Radiographics 1999;19:1107-24.

27. Sciubba JJ, Fantasia GE, Kahn LB.

Tumors ans cysts of the jaw. In: Rosai G, Sobin LH, editors. Atlas of tumors pathology. Washington: AFIP, 1999.

28. Bouchet $F$, Reynaud $P$, Borel J, Baudet-Pommel M, Dechelotte P. Une tumeur odontogène 
mandibulaire.

Ann Pathol 1999;19(2):145-6.

29. Guilbert F, Chomette $G$, Lecharpentier $\mathrm{Y}$,

Auriol M.

Les tumeurs bénignes et les pseudotumeurs

des maxillaires.

Rev Stomatol Chir

Maxillofac 1993;94:195-265.

30. Lezy GP, Princ G.

Pathologie maxillo-faciale et stomatologie.

Paris : Masson, 2004.

31. Kequeler JC, Lemay $\mathrm{O}$. Anatomie pathologique bucco-dentaire. Paris : Masson, 1998.

\section{SUMMARY}

\section{Complex odontoma: about a clinical case on mandible, complicated by an osteitis}

\author{
Hamid BOUKAIS, \\ Wacila ZERROUKI, \\ Chafika BABA-MEHDID, \\ Rachida AOUAMEUR, \\ Nadjah OUSSALEM, \\ Assia SLIMANI, \\ Assia HENNI, \\ Hocine SKLAB, \\ Kheira BOUZID-BENDISSARI
}

\section{Keywords}

- odontogenic tumors

- complex odontomas

- osteitis

- erupted

\section{Background}

Intra-osseous odontomas of the jaw may remain asymptomatic for a long time, but can sometimes be the aetiology of infection (cellulitis or osteitis). In scarce situations, these tumors can erupt in the oral cavity.

\section{Material and method}

Case study.

\section{Results}

A women patient aged 62 years consulted in our department for a recurrent mandibular infection. Clinical, radiological and histopathologic explorations revealed a complex odontoma in the mandible, complicated by osteitis.

\section{Conclusion}

Some similar cases have been reported in the world literature. Infection occurs when the odontoma tries to erupt in the oral cavity. When a recurrent infection happens in an edentelous sector of the jaw, radiological examinations must always be carried in order to discover the aetiology of this infection, wich can be an infected tumor as odontoma. 